\title{
On Blow-Up Structures for a Generalized Periodic Nonlinearly Dispersive Wave Equation
}

\author{
Ying Wang, ${ }^{1}$ Yunxi Guo, ${ }^{1,2}$ and Fang $\mathrm{Li}^{1}$ \\ ${ }^{1}$ Department of Applied Mathematics, Sichuan University of Science and Engineering, Zigong 643000, China \\ ${ }^{2}$ Sichuan Province University Key Laboratory of Bridge Non-Destruction Detecting and Engineering Computing, Zigong 643000, China \\ Correspondence should be addressed to Yunxi Guo; yunxigmaths@163.com
}

Received 6 November 2013; Accepted 23 February 2014; Published 7 April 2014

Academic Editor: Shaoyong Lai

Copyright (c) 2014 Ying Wang et al. This is an open access article distributed under the Creative Commons Attribution License, which permits unrestricted use, distribution, and reproduction in any medium, provided the original work is properly cited.

The local well-posedness for a generalized periodic nonlinearly dispersive wave equation is established. Under suitable assumptions on initial value $u_{0}$, a precise blow-up scenario and several sufficient conditions about blow-up results to the equation are presented.

\section{Introduction}

Recently, Hu and Yin [1] and Yin [2] investigate the following equation:

$$
u_{t}-u_{t x x}+2 \omega u_{x}+3 u u_{x}=\gamma\left(2 u_{x} u_{x x}+u u_{x x x}\right)
$$

where $\omega$ is nonnegative number and $\gamma$ is arbitrary real number. It is shown from $[1,2]$ that $(1)$ has solitary wave solutions and blow-up solutions for nonperiodic case and also solutions which blow up in finite time for periodic case.

If $\gamma=0$, (1) becomes the famous BBM equation modelling the motion of internal gravity waves in shallow channel [3]. Some results related to the equation can be found in $[4,5]$. It is worthwhile to mention that the equation does not have integrability and its solitary waves are not solitons [5].

If $\gamma=1$ in (1), attention is attracted to the wellknown Camassa-Holm equation, which models the unidirectional propagation of shallow water waves over a flat bottom. Here, $u(t, x)$ represents the free surface above a flat bottom and $\omega$ is a nonnegative parameter related to the critical shallow water speed [6]. As a model to describe the shallow water motion, the Camassa-Holm equation possesses a bi-Hamiltonian structure and infinite conservation laws [7-9] and is completely integrable [10]. It is regarded as a reexpression of geodesic flow on the diffeomorphism group of circle if $\omega=0$ [11] and on the Bott-Virasoro group if $\omega>0$ [12]. Recently, some significant results of dynamical behaviors have been obtained for the Cauchy problem of the CamassaHolm equation. For example, the local well-posedness of corresponding solution for initial data $u_{0} \in H^{s}(\mathbb{R})$ with $s>$ $3 / 2$ was given by several authors (see [13-15]). Under certain assumptions on initial data $u_{0}$, the equation has global strong solutions and blow-up solutions for periodic and nonperiodic case (see $[13,16-22])$. The existence and uniqueness of global weak solutions in $H^{1}(\mathbb{R})$ for the equation were proved (see [23-25]). It is shown from [6] that the solitary waves of the equation are peakon solitons and are orbitally stable.

If $\omega=0$ and $\gamma \in \mathbb{R},(1)$ changes into the rod equation derived by Dai [26] recently, which describes finitelength and small amplitude radial deformation waves in thin cylindrical compressible hyperelastic rods (see [26]), and $u(t, x)$ represents the radial stretch relative to a prestressed state in one-dimensional variable. The first investigation of the Cauchy problem of the rod equation on the line was done by Constantin and Strauss [27], the precise blow-up scenario, some blow-up results of strong solution, and the stability of a class of solitary waves to the rod equation are presented. In $[28,29]$, Zhou found the sufficient conditions to guarantee the finite blow-up of corresponding solution for periodic case. Moreover, Yin [30] discusses the rod equation on the circle and gives some interesting blow-up results. 
In this paper, we consider a generalized nonlinearly dispersive wave equation on the circle

$$
\begin{gathered}
u_{t}-u_{t x x}+2 \omega u_{x}+a u u_{x}+\beta\left(u-u_{x x}\right) \\
=\gamma\left(2 u_{x} u_{x x}+u u_{x x x}\right), \quad t>0, x \in \mathbb{R}, \\
u(0, x)=u_{0}(x), \quad x \in \mathbb{R}, \\
u(t, x+1)=u(t, x), \quad t>0, x \in \mathbb{R},
\end{gathered}
$$

where $\omega$ and $a$ are nonnegative fixed constants and $\beta$ and $\gamma$ are fixed arbitrary constants. Obviously, (2) reduces to (1) if we define $a=3$ and $\beta=0$. Actually, Wu and Yin [31] consider a nonlinearly dissipative Camassa-Holm equation which includes a nonlinearly dissipative term $L(u)$, where $L$ is a differential operator. Thus, we can regard the term $\beta\left(u-u_{x x}\right)$ as a dissipative term.

Because of the term $\beta\left(u-u_{x x}\right)$, (2) does not admit conservation laws in previous works $[1,2]$ :

$$
E(u)=\int_{\mathbb{S}}\left(u^{2}+u_{x}^{2}\right) d x
$$

Several estimates are established to prove several blowup solutions. More precisely, we establish the local wellposedness of strong solutions for (2) subject to initial value $u_{0} \in H^{r}(\mathbb{S}), r>3 / 2$, with $\mathbb{S}=\mathbb{R} / \mathbb{Z}$ (the circle of unit length) and give a precise blow-up scenario. Under suitable assumptions on the initial value $u_{0}$, relying on the classical mathematical techniques, the several sufficient conditions about blow-up solutions are found.

\section{Local Well-Posedness}

In this section, we establish the local well-posedness and blow-up scenario for the Cauchy problem (2) in $H^{r}(\mathbb{S}), r>$ $3 / 2$.

We denote by $*$ the convolution. Note that if $G(x):=$ $\cosh (x-[x]-1 / 2) / 2 \sinh (1 / 2)$, where $[x]$ stands for the integer part of $x \in R$, then $\left(1-\partial_{x}^{2}\right)^{-1} f=G * f$ for all $f \in L^{2}(\mathbb{R})$ and $G *\left(u-u_{x x}\right)=u$. Using this identity, (2) becomes

$$
\begin{gathered}
u_{t}+\gamma u u_{x}+\beta u+\partial_{x} G *\left[\frac{a-\gamma}{2} u^{2}+\frac{\gamma}{2}\left(u_{x}\right)^{2}+2 \omega u\right]=0 \\
t>0, x \in \mathbb{R} \\
u(0, x)=u_{0}(x), \\
u(t, x+1)=u(t, x),
\end{gathered}
$$

which is equivalent to

$$
\begin{aligned}
& y_{t}+\gamma u y_{x}+2 \gamma y u_{x}+2 \omega u_{x}+\beta y \\
& \quad+(a-3 \gamma) u u_{x}=0, \quad t>0, x \in \mathbb{R}, \\
& y(0, x)=u_{0}(x)-u_{0, x x}(x), \quad x \in \mathbb{R}, \\
& y(t, x)=y(t, x+1), \quad t>0, \quad x \in \mathbb{R} .
\end{aligned}
$$

Theorem 1. Given $u_{0} \in H^{r}(\mathbb{S})(r>3 / 2)$, there exist a maximal $T=T\left(a, b, \gamma, \omega, u_{0}\right)$ and a unique solution $u$ to problem (2), such that

$$
u=u\left(\cdot, u_{0}\right) \in C\left([0, T) ; H^{r}(\mathbb{S})\right) \bigcap C^{1}\left([0, T) ; H^{r-1}(\mathbb{S})\right) .
$$

Proof. The proof of Theorem 1 can be finished by using Kato's semigroup theory (see [1] or [2]). Here, we omit the detailed proof.

\section{Blow-Up Solutions}

Theorem 2. Let $u_{0} \in H^{r}(\mathbb{S}), r>3 / 2$; the solution of $u\left(\cdot, u_{0}\right)$ of problem (2) is uniformly bounded. Blow-up in finite time $T<$ $+\infty$ occurs if and only if

$$
\liminf _{t \uparrow T}\left\{\inf _{x \in S}\left[\gamma u_{x}(t, x)\right]\right\}=-\infty .
$$

Before proving the theorem, we give several useful lemmas.

Lemma 3 (Kato and Ponce [32]). If $r>0$, then $H^{r} \cap L^{\infty}$ is an algebra. Moreover

$$
\|u v\|_{H^{r}} \leq c\left(\|u\|_{L^{\infty}}\|v\|_{H^{r}}+\|u\|_{H^{r}}\|v\|_{L^{\infty}}\right),
$$

where $c$ is a constant depending only on $r$.

Lemma 4 (Kato and Ponce [32]). Let $r>0$. If $u \in H^{r} \cap W^{1, \infty}$ and $v \in H^{r-1} \cap L^{\infty}$, then

$$
\left\|\left[\Lambda^{r}, u\right] v\right\|_{L^{2}} \leq c\left(\left\|\partial_{x} u\right\|_{L^{\infty}}\left\|\Lambda^{r-1} v\right\|_{L^{2}}+\left\|\Lambda^{r} u\right\|_{L^{2}}\|v\|_{L^{\infty}}\right) .
$$

Lemma 5. Let $s \geq 3 / 2$ and $u(t, x)$ is the corresponding solution of (4) with initial data $u_{0}(x) \in H^{r}(\mathbb{S})$; it holds that if $q \in$ $(0, r-1]$, there is a constant $c$ depending only on q such that

$$
\begin{aligned}
\int_{\mathbb{S}}\left(\Lambda^{q+1} u\right)^{2} d x \leq & \int_{\mathbb{S}}\left(\Lambda^{q+1} u_{0}\right)^{2} d x \\
& +c \int_{0}^{t}\left(\left\|u_{x}\right\|_{L^{\infty}}+1\right)\|u\|_{H^{q+1}}^{2} d s .
\end{aligned}
$$

Proof. We rewrite (2) in the following equivalent form:

$$
\begin{aligned}
u_{t}-u_{t x x}= & -a u u_{x}-2 \omega u_{x}+\frac{\gamma}{2} \partial_{x}^{3} u^{2} \\
& -\frac{\gamma}{2} \partial_{x}\left(u_{x}^{2}\right)-\beta u+\beta \partial_{x}^{2} u .
\end{aligned}
$$


For $q \in(0, s-1]$, applying $\left(\Lambda^{q} u\right) \Lambda^{q}$ on both sides of (11) and integrating the new equation with respect to $x$ by parts, we obtain

$$
\begin{aligned}
& \frac{1}{2} \frac{d}{d t} \int_{\mathbb{S}}\left(\left(\Lambda^{q} u\right)^{2}+\left(\Lambda^{q} u_{x}\right)^{2}\right) d x \\
& =-a \int_{\mathbb{S}}\left(\Lambda^{q} u\right) \Lambda^{q}\left(u u_{x}\right)-\gamma \int_{\mathbb{S}}\left(\Lambda^{q+1} u\right) \Lambda^{q+1}\left(u u_{x}\right) \\
& \quad+\gamma \int_{\mathbb{S}}\left(\Lambda^{q} u\right) \Lambda^{q}\left(u u_{x}\right)+\frac{\gamma}{2} \int_{\mathbb{S}}\left(\Lambda^{q} u_{x}\right) \Lambda^{q}\left(u_{x}^{2}\right) \\
& \quad-\beta \int_{\mathbb{S}}\left(\Lambda^{q+1} u\right)^{2} d x
\end{aligned}
$$

where the Parseval equality

$$
\begin{aligned}
\int_{\mathbb{S}}\left(\Lambda^{q} u\right) \Lambda^{q} \partial_{x}^{3}\left(u^{2}\right) d x= & -2 \int_{S}\left(\Lambda^{q+1} u\right) \Lambda^{q+1}\left(u u_{x}\right) d x \\
& +2 \int_{\mathbb{S}}\left(\Lambda^{q} u\right) \Lambda^{q}\left(u u_{x}\right) d x
\end{aligned}
$$

is used. We will estimate each of the terms on the right-hand side of (11). For the first and the third terms, using integration by parts, the Cauchy-Schwartz inequality, and Lemmas 3 and 4 , we have

$$
\begin{aligned}
\int_{\mathbb{S}}\left(\Lambda^{q} u\right) \Lambda^{q}\left(u u_{x}\right) d x= & \int_{\mathbb{S}}\left(\Lambda^{q} u\right)\left[\Lambda^{q}\left(u u_{x}\right)-u \Lambda^{q} u_{x}\right] d x \\
& +\int_{\mathbb{S}}\left(\Lambda^{q} u\right) u \Lambda^{q}\left(u_{x}\right) d x \\
\leq & c\|u\|_{H^{q}}^{2}\left\|u_{x}\right\|_{L^{\infty}}+\frac{1}{2}\left\|u_{x}\right\|_{L^{\infty}}\left\|\Lambda^{q} u\right\|_{L^{2}}^{2} \\
\leq & c\|u\|_{H^{q}}^{2}\left\|u_{x}\right\|_{L^{\infty}}
\end{aligned}
$$

where $c$ only depends on $q$. Using the above estimate to the second term yields

$$
\int_{\mathbb{S}}\left(\Lambda^{q+1} u\right) \Lambda^{q+1}\left(u u_{x}\right) d x \leq c\|u\|_{H^{q+1}}^{2}\left\|u_{x}\right\|_{L^{\infty}}
$$

For the fourth term, using Lemma 3 gives rise to

$$
\begin{aligned}
\int_{\mathbb{S}}\left(\Lambda^{q} u_{x}\right) \Lambda^{q}\left(u_{x}^{2}\right) d x & \leq\left\|\Lambda^{q} u_{x}\right\|_{L^{2}}\left\|\Lambda^{q} u_{x}^{2}\right\|_{L^{2}} \\
& \leq c\|u\|_{H^{q+1}}\left(\left\|u_{x}\right\|_{L^{\infty}}\left\|u_{x}\right\|_{H^{q}}\right) \\
& \leq c\|u\|_{H^{q+1}}^{2}\left\|u_{x}\right\|_{L^{\infty}} .
\end{aligned}
$$

It follows from (12)-(16) that

$$
\frac{1}{2} \frac{d}{d t} \int_{\mathbb{S}}\left(\left(\Lambda^{q} u\right)^{2}+\left(\Lambda^{q} u_{x}\right)^{2}\right) d x \leq c\left(\left\|\gamma u_{x}\right\|_{L^{\infty}}+1\right)\|u\|_{H^{q+1}}^{2},
$$

which leads to

$$
\begin{aligned}
& \int_{\mathbb{S}}\left(\left(\Lambda^{q} u\right)^{2}+\left(\Lambda^{q} u_{x}\right)^{2}\right) d x-\int_{\mathbb{S}}\left(\left(\Lambda^{q} u_{0}\right)^{2}+\left(\Lambda^{q} u_{0 x}\right)^{2}\right) d x \\
& \quad \leq c \int_{0}^{t}\left(\left\|\gamma u_{x}\right\|_{L^{\infty}}+1\right)\|u\|_{H^{q+1}}^{2} d s
\end{aligned}
$$

It completes the proof of the lemma.
Proof of Theorem 2. Applying (10) with $q=r-1$, we have

$$
\|u\|_{H^{r}(\mathbb{S})}^{2} \leq\left\|u_{0}\right\|_{H^{r}(\mathbb{S})}^{2}+c \int_{0}^{t}\left(\left\|\gamma u_{x}\right\|_{L^{\infty}(\mathbb{S})}+1\right)\|u\|_{H^{r}(\mathbb{S})}^{2} d s .
$$

It follows from (19) and Gronwall's inequality that

$$
\|u\|_{H^{r}(\mathbb{S})}^{2} \leq\left\|u_{0}\right\|_{H^{r}(\mathbb{S})}^{2} \exp \left(c \int_{0}^{t}\left(\left\|\gamma u_{x}\right\|_{L^{\infty}(\mathbb{S})}+1\right) d s\right) .
$$

If there is a constant $M>0$ such that $\left\|\gamma u_{x}\right\|_{L^{\infty}} \leq M$ on $(0, T]$, then $\|u\|_{H^{r}(\mathbb{S})}^{2}$ does not blow up. It completes the proof of Theorem 2.

Lemma 6 (see [17]). Let $u(t)$ be the solution to (2) on $[0, T)$ with initial data $u_{0} \in H^{s}(\mathbb{S}), s>3 / 2$. Then there exists at least one point $\xi(t) \in \mathbb{S}$ with

$$
m(t)=\inf _{x \in \mathbb{S}}\left[u_{x}(x, t)\right]=u_{x}(t, \xi(t)) .
$$

The function $m(t)$ is almost everywhere differentiable on $(0, t)$ with

$$
\frac{d m(t)}{d t}=u_{t x}(t,(\xi(t)))
$$

Lemma 7 (see $[21,22])$. (i) For every $u \in H^{1}(\mathbb{S})$, one has

$$
\max _{x \in[0,1]} u^{2}(x) \leq \frac{e+1}{2(e-1)}\|u\|_{H^{1}(\mathbb{S})}^{2},
$$

where the constant $(e+1) / 2(e-1)$ is sharp.

(ii) For every $u \in H^{3}(\mathbb{S})$, one has

$$
\max _{x \in[0,1]} u^{2}(x) \leq c\|u\|_{H^{1}(\mathbb{S})}^{2}
$$

with the best possible constant $c$ lying within the range $(1,13 / 12]$. Moreover, the best constant $c$ is $(e+1) / 2(e-1)$.

Lemma 8 (see [2]). If $f \in H^{3}(\mathbb{S})$ is such that $\int_{\mathbb{S}} f(x) d x=$ $a_{0} / 2$, then, for every $\varepsilon>0$, one has

$$
\max _{x \in[0,1]} f^{2}(x) \leq \frac{\varepsilon+2}{24} \int_{\mathbb{S}} f_{x}^{2} d x+\frac{\varepsilon+2}{4 \varepsilon} a_{0}^{2} .
$$

Moreover,

$$
\max _{x \in[0,1]} f^{2}(x) \leq \frac{\varepsilon+2}{24}\|f\|_{H^{1}}^{2}+\frac{\varepsilon+2}{4 \varepsilon} a_{0}^{2} .
$$

Lemma 9. Let $u \in H^{3}(\mathbb{S})$ and $T>0$ be the maximal existence time of the solution $u(t, x)$ to problem (4). Then it holds that

(i) $\int_{\mathbb{S}} u(t, x) d x=\int_{\mathbb{S}} u_{0}(x) d x=\int_{\mathbb{S}} y(t, x) d x=$
$\int_{\mathbb{S}} y_{0}(x) d x$,

(ii) $\|u\|_{H^{1}}^{2}=\|u\|_{H^{1}}^{2} e^{-2 \beta t}$. 
Proof. The proof of (i) is similar to that of [2, lemma 3.6], so we omit it.

Multiplying $u$ to both sides of (2) and integrating by parts, we get

$$
\frac{1}{2} \frac{d}{d t} \int_{\mathbb{S}}\left(u^{2}+u_{x}^{2}\right) d x=-\beta \int_{\mathbb{S}}\left(u^{2}+u_{x}^{2}\right) d x
$$

which yields

$$
\|u\|_{H^{1}(\mathbb{S})}^{2}=\left\|u_{0}\right\|_{H^{1}(\mathbb{S})}^{2} e^{-2 \beta t}
$$

It finishes the proof.

Lemma 10 (see [29]). Assume that a differential function $y(t)$ satisfies

$$
y^{\prime} \leq-C y^{2}(t)+K
$$

with constants $C, K>0$. If the initial datum $y(0)=y_{0}<$ $-\sqrt{K / C}$, then the solutions to (29) go to $-\infty$ in finite time.

We now present the first blow-up result.

Theorem 11. Let $\beta<0$ and $u_{0} \in H^{s}(\mathbb{S}), s \geq 3$.

(i) If $0<\gamma<a / 3$ and there is a $x_{0} \in \mathbb{S}$ such that

$$
\begin{aligned}
\gamma u_{0}^{\prime}\left(x_{0}\right)<-\beta-\sqrt{2}\left(\frac{\beta^{2}}{2}\right. & +\frac{\gamma(a-2 \gamma)(e+1)}{4(e-1)}\left\|u_{0}\right\|_{H^{1}}^{2} e^{2 \beta T} \\
& \left.+4 \gamma \omega \sqrt{\frac{e+1}{2(e-1)}}\left\|u_{0}\right\|_{H^{1}} e^{\beta T}\right)^{1 / 2},
\end{aligned}
$$

then the corresponding solution to (2) blows up in finite time.

(ii) If $a / 3<\gamma<a$ and there is a $x_{0} \in \mathbb{S}$ such that

$$
\begin{array}{r}
\gamma u_{0}^{\prime}\left(x_{0}\right)<-\beta-\sqrt{2}\left(\frac{\beta^{2}}{2}+\frac{\gamma(a-\gamma)(e+1)}{8(e-1)}\left\|u_{0}\right\|_{H^{1}}^{2} e^{2 \beta T}\right. \\
\left.+4 \gamma \omega \sqrt{\frac{e+1}{2(e-1)}}\left\|u_{0}\right\|_{H^{1}} e^{\beta T}\right)^{1 / 2}
\end{array}
$$

then the corresponding solution to (2) blows up in finite time.

(iii) If $\gamma<0$ or $\gamma>a$ and there is a $x_{0} \in \mathbb{S}$ such that

$$
\begin{aligned}
& \gamma u_{0}^{\prime}\left(x_{0}\right)<-\beta-\sqrt{2}( \frac{\beta^{2}}{2}+\frac{\gamma(\gamma-a)(e+1)}{4(e-1)}\left\|u_{0}\right\|_{H^{1}}^{2} e^{2 \beta T} \\
&\left.+4|\gamma| \omega \sqrt{\frac{e+1}{2(e-1)}}\left\|u_{0}\right\|_{H^{1}} e^{\beta T}\right)^{1 / 2},
\end{aligned}
$$

then the corresponding solution to (2) blows up in finite time.
Proof. Let $T>0$ be the maximal time of existence of the solution $u$ to (2) with the initial data $u_{0}$. Note that $\partial_{x}^{2} G * f=$ $G * f-f$. Differentiating (4) with respect to $x$ and multiplying the obtained equation by $\gamma$, we get

$$
\begin{aligned}
\gamma u_{t x}= & -\frac{\gamma^{2}}{2} u_{x}^{2}-\gamma^{2} u u_{x x}+\frac{\gamma(a-\gamma)}{2} u^{2}+2 \gamma \omega u-\gamma \beta u_{x} \\
& -G *\left[\frac{(a-\gamma) \gamma}{2} u^{2}+\frac{\gamma^{2}}{2} u_{x}^{2}+2 \gamma \omega u\right] .
\end{aligned}
$$

Noting that $u_{x x}(t, \xi(t))=0$, for all $t \in[0, T)$, and defining $m(t)=\gamma u_{x}(t, \xi(t))+\beta=\inf _{x \in \mathbb{S}}\left(\gamma u_{x}(t, x)+\beta\right)$, we obtain

$$
\begin{aligned}
m^{\prime}(t)= & -\frac{1}{2} m^{2}(t)+\frac{\beta^{2}}{2}+\frac{\gamma(a-\gamma)}{2} u^{2}+2 \gamma \omega u \\
& -G *\left[\frac{(a-\gamma) \gamma}{2} u^{2}+\frac{\gamma^{2}}{2} u_{x}^{2}+2 \gamma \omega u\right] .
\end{aligned}
$$

Next, we divide (34) into three cases to prove the theorem.

(i) The first is $0<\gamma<a / 3$; note that $(a-3 \gamma) \gamma / 2 \geq 0$ and $G *\left(u^{2}+(1 / 2) u_{x}^{2}\right) \geq(1 / 2) u^{2}$. Then, we have

$$
\begin{array}{rl}
G & *\left[\frac{(a-\gamma) \gamma}{2} u^{2}+\frac{\gamma^{2}}{2} u_{x}^{2}\right] \\
& =G *\left[\gamma^{2} u^{2}+\frac{\gamma^{2}}{2} u_{x}^{2}+\left(\frac{(a-\gamma) \gamma}{2}-\gamma^{2}\right) u^{2}\right] \\
& \geq \frac{\gamma^{2}}{2} u^{2}+G *\left(\frac{(a-3 \gamma) \gamma}{2} u^{2}\right) .
\end{array}
$$

Note that $G *\left(((a-3 \gamma) \gamma / 2) u^{2}\right) \geq 0$. Thus, we obtain

$$
\begin{aligned}
m^{\prime}(t) \leq & -\frac{1}{2} m^{2}(t)+\frac{\beta^{2}}{2}+\frac{\gamma(a-2 \gamma)}{2} u^{2} \\
& +2 \gamma \omega u-G *(2 \gamma \omega u) .
\end{aligned}
$$

From Lemmas 8 and 9, we have

$$
\max _{x \in \mathbb{S}}\left\{u^{2}\right\} \leq \frac{e+1}{2(e-1)}\|u\|_{H^{1}}^{2} \leq \frac{e+1}{2(e-1)}\left\|u_{0}\right\|_{H^{1}}^{2} e^{-2 \beta T} .
$$

Thus,

$$
\|u\|_{L^{\infty}} \leq \sqrt{\frac{e+1}{2(e-1)}}\left\|u_{0}\right\|_{H^{1}} e^{-\beta T} .
$$

Note that $\|G\|_{L^{1}(\mathbb{S})}=1$. Using Young's inequality, we get

$$
|G * u| \leq\|u\|_{L^{\infty}}\|G\|_{L^{1}(\mathbb{S})} \leq \sqrt{\frac{e+1}{2(e-1)}}\left\|u_{0}\right\|_{H^{1}} e^{-\beta T} .
$$

Therefore, we deduce

$$
\begin{aligned}
m^{\prime}(t) \leq & -\frac{1}{2} m^{2}(t)+\frac{\beta^{2}}{2}+\frac{\gamma(a-2 \gamma)}{2} \frac{e+1}{2(e-1)} \\
& \times\left\|u_{0}\right\|_{H^{1}}^{2} e^{-2 \beta T}+4 \gamma \omega \sqrt{\frac{e+1}{2(e-1)}}\left\|u_{0}\right\|_{H^{1}} e^{-\beta T},
\end{aligned}
$$


which results in

$$
m^{\prime}(t) \leq-\frac{1}{2} m^{2}(t)+K
$$

with

$$
\begin{aligned}
K= & \frac{\beta^{2}}{2}+\frac{\gamma(a-2 \gamma)}{2} \frac{e+1}{2(e-1)}\left\|u_{0}\right\|_{H^{1}}^{2} e^{-2 \beta T} \\
& +4 \gamma \omega \sqrt{\frac{e+1}{2(e-1)}}\left\|u_{0}\right\|_{H^{1}} e^{-\beta T} .
\end{aligned}
$$

Note that, from Lemma 10 , if $m(0)<-\sqrt{2 K}$, then there exists $T$, such that $\lim _{t \uparrow T} m(t)=-\infty$. Applying Theorem 2, the solution $u$ to (2) does not exist globally in time.

(ii) The second is $a / 3 \leq \gamma \leq a$. Similar to case (i), we have $\gamma(3 \gamma-a) / 4 \geq 0$ and

$$
\begin{aligned}
G *\left[\frac{(a-\gamma) \gamma}{2} u^{2}+\frac{\gamma^{2}}{2} u_{x}^{2}\right] & \geq \frac{(a-\gamma) \gamma}{4} u^{2} \\
& +G *\left(\frac{\gamma(3 \gamma-a)}{4} u_{x}^{2}\right) .
\end{aligned}
$$

Therefore, we obtain

$$
\begin{aligned}
m^{\prime}(t) \leq & -\frac{1}{2} m^{2}(t)+\frac{\beta^{2}}{2}+\frac{\gamma(a-\gamma)}{4} u^{2} \\
& +2 \gamma \omega u-G *(2 \gamma \omega u) .
\end{aligned}
$$

Using (37)-(39), we get

$$
\begin{aligned}
m^{\prime}(t) \leq & -\frac{1}{2} m^{2}(t)+\frac{\beta^{2}}{2}+\frac{\gamma(a-\gamma)}{4} \frac{e+1}{2(e-1)} \\
& \times\left\|u_{0}\right\|_{H^{1}}^{2} e^{-2 \beta T}+4 \gamma \omega \sqrt{\frac{e+1}{2(e-1)}}\left\|u_{0}\right\|_{H^{1}} e^{-\beta T},
\end{aligned}
$$

which results in

$$
m^{\prime}(t) \leq-\frac{1}{2} m^{2}(t)+K
$$

with

$$
\begin{aligned}
K= & \frac{\beta^{2}}{2}+\frac{\gamma(a-\gamma)}{4} \frac{e+1}{2(e-1)}\left\|u_{0}\right\|_{H^{1}}^{2} e^{-2 \beta T} \\
& +4 \gamma \omega \sqrt{\frac{e+1}{2(e-1)}}\left\|u_{0}\right\|_{H^{1}} e^{-\beta T} .
\end{aligned}
$$

Note that, from Lemma 10 , if $m(0)<-\sqrt{2 K}$, then there exists $T$, such that $\lim _{t \uparrow T} m(t)=-\infty$. Repeating the proof of (i), we conclude that the solution $u$ blows up in finite time.

(iii) The third is $\gamma<0$ or $\gamma>a$; note that $(a-\gamma) \gamma / 2<0$. From Young's inequality, we get for all $t \in[0, T)$

$$
\left|G * u^{2}\right| \leq\|u\|_{L^{\infty}}^{2}\|G\|_{L^{1}} \leq \frac{e+1}{2(e-1)}\left\|u_{0}\right\|_{H^{1}}^{2} e^{-2 \beta T} .
$$

Using (37)-(39), we get

$$
\begin{aligned}
m^{\prime}(t) \leq & -\frac{1}{2} m^{2}(t)+\frac{\beta^{2}}{2}+\frac{\gamma(\gamma-a)}{2} \frac{e+1}{2(e-1)}\left\|u_{0}\right\|_{H^{1}}^{2} e^{-2 \beta T} \\
& +4|\gamma| \omega \sqrt{\frac{e+1}{2(e-1)}}\left\|u_{0}\right\|_{H^{1}} e^{-\beta T}
\end{aligned}
$$

which results in

$$
m^{\prime}(t) \leq-\frac{1}{2} m^{2}(t)+K,
$$

with

$$
\begin{aligned}
K= & \frac{\beta^{2}}{2}+\frac{\gamma(\gamma-a)}{2} \frac{e+1}{2(e-1)}\left\|u_{0}\right\|_{H^{1}}^{2} e^{-2 \beta T} \\
& +4|\gamma| \omega \sqrt{\frac{e+1}{2(e-1)}}\left\|u_{0}\right\|_{H^{1}} e^{-\beta T} .
\end{aligned}
$$

Note that, from Lemma 10 , if $m(0)<-\sqrt{2 K}$, then there exists $T$, such that $\lim _{t \uparrow T} m(t)=-\infty$. Repeating the proof of (i), we deduce that the solution $u$ blows up in finite time. It finishes the proof of the theorem.

Next, we give the second blow-up result.

Theorem 12. Let $\beta<0$ and $u_{0} \in H^{s}(\mathbb{S}), s \geq 3$, and $\int_{\mathbb{S}} u_{0} d x=$ $a_{0} / 2$.

(i) If $0<\gamma<a / 3$ and for all $\epsilon>0$ there is a $x_{0} \in \mathbb{S}$ such that

$$
\begin{aligned}
& \gamma u_{0}^{\prime}\left(x_{0}\right) \\
&<-\beta-\sqrt{2}\left(\frac{\beta^{2}}{2}+\frac{\gamma(a-2 \gamma)}{2}\right. \\
& \times\left(\frac{\epsilon+2}{24}\left\|u_{0}\right\|_{H^{1}}^{2} e^{-2 \beta T}+\frac{\epsilon+2}{4 \epsilon} a_{0}^{2}\right)+4 \gamma \omega \\
&\left.\times \sqrt{\frac{\epsilon+2}{24}\left\|u_{0}\right\|_{H^{1}} e^{-2 \beta T}+\frac{\epsilon+2}{4 \epsilon} a_{0}^{2}}\right)^{1 / 2},
\end{aligned}
$$

then the corresponding solution to (2) blows up in finite time.

(ii) If $a / 3<\gamma<a$ and for all $\epsilon>0$ there is a $x_{0} \in \mathbb{S}$ such that

$$
\begin{aligned}
& \gamma u_{0}^{\prime}\left(x_{0}\right)<-\beta-\sqrt{2}\left(\frac{\beta^{2}}{2}+\frac{\gamma(a-\gamma)}{4}\left(\frac{\epsilon+2}{24}\left\|u_{0}\right\|_{H^{1}}^{2} e^{-2 \beta T}\right.\right.\left.+\frac{\epsilon+2}{4 \epsilon} a_{0}^{2}\right)+4 \gamma \omega \\
&\left.\times \sqrt{\frac{\epsilon+2}{24}\left\|u_{0}\right\|_{H^{1}} e^{-2 \beta T}+\frac{\epsilon+2}{4 \epsilon} a_{0}^{2}}\right)^{1 / 2},
\end{aligned}
$$

then the corresponding solution to (2) blows up in finite time. 
(iii) If $\gamma<0$ or $\gamma>a$ and for all $\epsilon>0$ there is a $x_{0} \in \mathbb{S}$ such that

$$
\begin{array}{r}
\gamma u_{0}^{\prime}\left(x_{0}\right)<-\beta-\sqrt{2}\left(\frac{\beta^{2}}{2}+\frac{\gamma(\gamma-a)}{2}\left(\frac{\epsilon+2}{24}\left\|u_{0}\right\|_{H^{1}}^{2} e^{-2 \beta T}\right.\right. \\
\left.+\frac{\epsilon+2}{4 \epsilon} a_{0}^{2}\right)+4|\gamma| \omega \\
\left.\times \sqrt{\frac{\epsilon+2}{24}\left\|u_{0}\right\|_{H^{1}} e^{-2 \beta T}+\frac{\epsilon+2}{4 \epsilon} a_{0}^{2}}\right)^{1 / 2},
\end{array}
$$

then the corresponding solution to (2) blows up in finite time.

Proof. Similar to Theorem 11, we divide (34) into three cases to prove the theorem.

(i) The first is $0<\gamma<a / 3$; note that $(a-3 \gamma) \gamma / 2 \geq 0$ and $G *\left(u^{2}+(1 / 2) u_{x}^{2}\right) \geq(1 / 2) u^{2}$. Then, we have $G *((a-$ $3 \gamma) \gamma / 2) u^{2} \geq 0$.

From Lemmas 8 and 9, we have

$$
\begin{aligned}
\max _{x \in S}\left\{u^{2}\right\} & \leq \frac{\epsilon+2}{24}\|u\|_{H^{1}}^{2}+\frac{\epsilon+2}{4 \epsilon} a_{0}^{2} \\
& \leq \frac{\epsilon+2}{24}\left\|u_{0}\right\|_{H^{1}}^{2} e^{-2 \beta T}+\frac{\epsilon+2}{4 \epsilon} a_{0}^{2} .
\end{aligned}
$$

Thus,

$$
\|u\|_{L^{\infty}} \leq \sqrt{\frac{\epsilon+2}{24}\left\|u_{0}\right\|_{H^{1}} e^{-2 \beta T}+\frac{\epsilon+2}{4 \epsilon} a_{0}^{2}} .
$$

Note that $\|G\|_{L^{1}(\mathbb{S})}=1$. Using Young's inequality, we get

$$
\begin{aligned}
|G * u| & \leq\|u\|_{L^{\infty}}\|G\|_{L^{1}(\mathbb{S})} \\
& \leq \sqrt{\frac{\epsilon+2}{24}\left\|u_{0}\right\|_{H^{1}} e^{-2 \beta T}+\frac{\epsilon+2}{4 \epsilon} a_{0}^{2}} .
\end{aligned}
$$

Therefore, we deduce

$$
\begin{aligned}
m^{\prime}(t) \leq & -\frac{1}{2} m^{2}(t)+\frac{\beta^{2}}{2}+\frac{\gamma(a-2 \gamma)}{2} \\
& \times\left(\frac{\epsilon+2}{24}\left\|u_{0}\right\|_{H^{1}}^{2} e^{-2 \beta T}+\frac{\epsilon+2}{4 \epsilon} a_{0}^{2}\right) \\
& +4 \gamma \omega \sqrt{\frac{\epsilon+2}{24}\left\|u_{0}\right\|_{H^{1}} e^{-2 \beta T}+\frac{\epsilon+2}{4 \epsilon} a_{0}^{2}},
\end{aligned}
$$

which results in

$$
m^{\prime}(t) \leq-\frac{1}{2} m^{2}(t)+K
$$

with

$$
\begin{aligned}
K= & \frac{\beta^{2}}{2}+\frac{\gamma(a-2 \gamma)}{2}\left(\frac{\epsilon+2}{24}\left\|u_{0}\right\|_{H^{1}}^{2} e^{-2 \beta T}+\frac{\epsilon+2}{4 \epsilon} a_{0}^{2}\right) \\
& +4 \gamma \omega \sqrt{\frac{\epsilon+2}{24}\left\|u_{0}\right\|_{H^{1}} e^{-2 \beta T}+\frac{\epsilon+2}{4 \epsilon} a_{0}^{2}} .
\end{aligned}
$$

Note that, from the lemma, if $m(0)<-\sqrt{2 K}$, then there exists $T$, such that $\lim _{t \uparrow T} m(t)=-\infty$. Applying Theorem 2, the solution $u$ blows up in finite time.

(ii) The second is $a / 3 \leq \gamma \leq a$. Similar to case (i), we have $\gamma(3 \gamma-a) / 4 \geq 0$ and

$$
\begin{aligned}
G *\left[\frac{(a-\gamma) \gamma}{2} u^{2}+\frac{\gamma^{2}}{2} u_{x}^{2}\right] \geq & \frac{(a-\gamma) \gamma}{4} u^{2} \\
& +G *\left(\frac{\gamma(3 \gamma-a)}{4} u_{x}^{2}\right) .
\end{aligned}
$$

Using (55)-(57), we get

$$
\begin{aligned}
m^{\prime}(t) \leq & -\frac{1}{2} m^{2}(t)+\frac{\beta^{2}}{2}+\frac{\gamma(a-\gamma)}{4} \\
& \times\left(\frac{\epsilon+2}{24}\left\|u_{0}\right\|_{H^{1}}^{2} e^{-2 \beta T}+\frac{\epsilon+2}{4 \epsilon} a_{0}^{2}\right) \\
& +4 \gamma \omega \sqrt{\frac{\epsilon+2}{24}\left\|u_{0}\right\|_{H^{1}} e^{-2 \beta T}+\frac{\epsilon+2}{4 \epsilon} a_{0}^{2}},
\end{aligned}
$$

which results in

$$
m^{\prime}(t) \leq-\frac{1}{2} m^{2}(t)+K
$$

with

$$
\begin{aligned}
K= & \frac{\beta^{2}}{2}+\frac{\gamma(a-\gamma)}{4} \frac{\epsilon+2}{24}\left\|u_{0}\right\|_{H^{1}}^{2} e^{-2 \beta T} \\
& +\frac{\epsilon+2}{4 \epsilon} a_{0}^{2}+4 \gamma \omega \sqrt{\frac{\epsilon+2}{24}\left\|u_{0}\right\|_{H^{1}} e^{-2 \beta T}+\frac{\epsilon+2}{4 \epsilon} a_{0}^{2}} .
\end{aligned}
$$

Following the proof of (i) in Theorem 11, we derive that the solution $u$ blows up in finite time.

(iii) The third is $\gamma<0$ or $\gamma>a$; note that $(a-\gamma) \gamma / 2<0$. From Young's inequality, we get for all $t \in[0, T)$

$$
\left|G * u^{2}\right| \leq\|u\|_{L^{\infty}}^{2}\|G\|_{L^{1}} \leq \frac{\epsilon+2}{24}\left\|u_{0}\right\|_{H^{1}}^{2} e^{-2 \beta T}+\frac{\epsilon+2}{4 \epsilon} a_{0}^{2} .
$$

Using (55)-(57) and (65), we get

$$
\begin{aligned}
m^{\prime}(t) \leq & -\frac{1}{2} m^{2}(t)+\frac{\beta^{2}}{2}+\frac{\gamma(\gamma-a)}{2} \\
& \times\left(\frac{\epsilon+2}{24}\left\|u_{0}\right\|_{H^{1}}^{2} e^{-2 \beta T}+\frac{\epsilon+2}{4 \epsilon} a_{0}^{2}\right) \\
& +4|\gamma| \omega \sqrt{\frac{\epsilon+2}{24}\left\|u_{0}\right\|_{H^{1}} e^{-2 \beta T}+\frac{\epsilon+2}{4 \epsilon} a_{0}^{2}},
\end{aligned}
$$

which results in

$$
m^{\prime}(t) \leq-\frac{1}{2} m^{2}(t)+K
$$

with

$$
\begin{aligned}
K= & \frac{\beta^{2}}{2}+\frac{\gamma(\gamma-a)}{2} \frac{\epsilon+2}{24}\left\|u_{0}\right\|_{H^{1}}^{2} e^{-2 \beta T} \\
& +\frac{\epsilon+2}{4 \epsilon} a_{0}^{2}+4|\gamma| \omega \sqrt{\frac{\epsilon+2}{24}\left\|u_{0}\right\|_{H^{1}} e^{-2 \beta T}+\frac{\epsilon+2}{4 \epsilon} a_{0}^{2} .}
\end{aligned}
$$


Following the proof of (i) in Theorem 11, we obtain that the solution $u$ blows up in finite time. It finishes the proof of the theorem.

Next, we give the third blow-up result.

Theorem 13. Assume that $\beta<0$ and $u_{0} \in H^{s}(\mathbb{S}), s \geq 3$, and $\left\|u_{0}\right\|_{H^{1}} \neq 0$.

(i) If $0<\gamma<a / 3$ is such that

$$
\begin{aligned}
\int_{\mathbb{S}} \gamma u_{0 x}^{3} d x<-2 \beta\left\|u_{0}\right\|_{H^{1}}^{2} e^{-2 \beta T} & \\
-\sqrt{\frac{2}{3}} & \left(\frac{\beta^{2}}{2}\left\|u_{0}\right\|_{H^{1}}^{4} e^{-4 \beta T}+\frac{3 \gamma(a-2 \gamma)(e+1)}{4(e-1)}\right. \\
& \times\left\|u_{0}\right\|_{H^{1}}^{6} e^{-6 \beta T}+12 \omega \gamma \\
& \left.\times \sqrt{\frac{e+1}{2(e-1)}}\left\|u_{0}\right\|_{H^{1}}^{5} e^{-5 \beta T}\right)^{1 / 2}
\end{aligned}
$$

then the corresponding solution to (2) blows up in finite time.

(ii) If $a / 3<\gamma<a$ is such that

$$
\begin{aligned}
\int_{\mathbb{S}} \gamma u_{0 x}^{3} d x<-2 \beta\left\|u_{0}\right\|_{H^{1}}^{2} e^{-2 \beta T} & -\sqrt{\frac{2}{3}}\left(\frac{\beta^{2}}{2}\left\|u_{0}\right\|_{H^{1}}^{4} e^{-4 \beta T}+\frac{3 \gamma(a-\gamma)(e+1)}{8(e-1)}\right. \\
& \times\left\|u_{0}\right\|_{H^{1}}^{6} e^{-6 \beta T}+12 \omega \gamma \\
& \left.\times \sqrt{\frac{e+1}{2(e-1)}}\left\|u_{0}\right\|_{H^{1}}^{5} e^{-5 \beta T}\right)^{1 / 2},
\end{aligned}
$$

then the corresponding solution to (2) blows up in finite time.

(iii) If $\gamma>$ a or $\gamma<0$ is such that

$$
\begin{aligned}
\int_{\mathbb{S}} \gamma u_{0 x}^{3} d x<-2 \beta\left\|u_{0}\right\|_{H^{1}}^{2} e^{-2 \beta T} & -\sqrt{\frac{2}{3}}\left(\frac{\beta^{2}}{2}\left\|u_{0}\right\|_{H^{1}}^{4} e^{-4 \beta T}+\frac{3 \gamma(\gamma-a)(e+1)}{8(e-1)}\right. \\
& \times\left\|u_{0}\right\|_{H^{1}}^{6} e^{-6 \beta T}+12 \omega|\gamma| \\
& \left.\times \sqrt{\frac{e+1}{2(e-1)}}\left\|u_{0}\right\|_{H^{1}}^{5} e^{-5 \beta T}\right)^{1 / 2}
\end{aligned}
$$

then the corresponding solution to (2) blows up in finite time.
Proof. Let $T>0$ be the maximal time of existence of the solution $u$ to (2) with the initial data $u_{0}$. Applying $\gamma u_{x}^{2} \partial_{x}$ to both sides of (2) and integrating by parts, we get

$$
\begin{aligned}
\frac{d}{d t} \int_{\mathbb{S}} \gamma u_{x}^{3} d x= & -\frac{3 \gamma^{2}}{2} \int_{\mathbb{S}} u_{x}^{4}-3 \beta \int_{\mathbb{S}} \gamma u_{x}^{3} d x+3 \frac{(a-\gamma) \gamma}{2} \\
& \times \int_{\mathbb{S}} u_{x}^{2} u^{2} d x+6 \omega \gamma \int_{\mathbb{S}} u_{x}^{2} u d x+3 \\
& \times \int_{\mathbb{S}} u_{x}^{2} G *\left(\frac{(a-\gamma) \gamma}{2} u^{2}+\frac{\gamma^{2}}{2} u_{x}^{2}+2 \omega \gamma u\right) .
\end{aligned}
$$

Since

$$
\left|\int_{\mathbb{S}} u_{x}^{3} d x\right| \leq\left(\int_{\mathbb{S}} u_{x}^{4} d x\right)^{1 / 2}\left(\int_{\mathbb{S}} u_{x}^{2} d x\right)^{1 / 2}
$$

thus

$$
\int_{\mathbb{S}} u_{x}^{4} d x \geq \frac{\left(\int_{\mathbb{S}} u_{x}^{3} d x\right)^{2}}{\int_{\mathbb{S}} u_{x}^{2} d x} \geq \frac{\left(\int_{\mathbb{S}} u_{x}^{3} d x\right)^{2}}{\|u\|_{H^{1}}^{2}}
$$

Therefore, we obtain

$$
\begin{aligned}
\frac{d}{d t} \int_{\mathbb{S}} \gamma u_{x}^{3} d x \leq & -\frac{3}{2\left\|u_{0}\right\|_{H^{1}}^{2} e^{-2 \beta T}}\left(\int_{\mathbb{S}} \gamma u_{x}^{3} d x\right)^{2}-3 \beta \int_{\mathbb{S}} \gamma u_{x}^{3} d x \\
& +3 \frac{(a-\gamma) \gamma}{2} \int_{\mathbb{S}} u_{x}^{2} u^{2} d x+6 \omega \gamma \int_{\mathbb{S}} u_{x}^{2} u d x \\
& +3 \int_{\mathbb{S}} u_{x}^{2} G *\left(\frac{(a-\gamma) \gamma}{2} u^{2}\right. \\
& \left.+\frac{\gamma^{2}}{2} u_{x}^{2}+2 \omega \gamma u\right) d x .
\end{aligned}
$$

Next, we divide (75) into three cases to prove the theorem.

(i) The first is $0<\gamma \leq a / 3$; from case (i) of Theorem 11, we know that $\gamma(a-3 \gamma) \geq 0$ and $G *\left(((a-3 \gamma) \gamma / 2) u^{2}\right) \geq 0$.

From Holder's inequality and Yong's inequality, we have

$$
\begin{aligned}
\left|\int_{\mathbb{S}} u_{x}^{2} u^{2} d x\right| & \leq\|u\|_{L^{\infty}}^{2} \int_{\mathbb{S}} u_{x}^{2} d x \leq \frac{e+1}{2(e-1)}\|u\|_{H^{1}}^{4} \\
& \leq \frac{e+1}{2(e-1)}\left\|u_{0}\right\|_{H^{1}}^{4} e^{-4 \beta T} \\
\left|\int_{\mathbb{S}} u_{x}^{2} u d x\right| & \leq\|u\|_{L^{\infty}} \int_{\mathbb{S}} u_{x}^{2} d x \leq \sqrt{\frac{e+1}{2(e-1)}}\|u\|_{H^{1}}^{3} \\
& \leq \sqrt{\frac{e+1}{2(e-1)}}\left\|u_{0}\right\|_{H^{1}}^{3} e^{-3 \beta T}
\end{aligned}
$$




$$
\begin{aligned}
\left|\int_{\mathbb{S}} u_{x}^{2} G * u d x\right| & \leq\|G * u\|_{L^{\infty}} \int_{\mathbb{S}} u_{x}^{2} d x \leq \sqrt{\frac{e+1}{2(e-1)}}\|u\|_{H^{1}}^{3} \\
& \leq \sqrt{\frac{e+1}{2(e-1)}}\left\|u_{0}\right\|_{H^{1}}^{3} e^{-3 \beta T} .
\end{aligned}
$$

Using (76) and (75), it yields

$$
\begin{aligned}
\frac{d}{d t} \int_{\mathbb{S}} \gamma u_{x}^{3} d x \leq & -\frac{3}{2\left\|u_{0}\right\|_{H^{1}}^{2} e^{-2 \beta T}} \\
& \times\left(\int_{\mathbb{S}} \gamma u_{x}^{3} d x+\beta\left\|u_{0}\right\|_{H^{1}}^{2} e^{-2 \beta T}\right)^{2}+\frac{3 \beta^{2}}{2} \\
& \times\left\|u_{0}\right\|_{H^{1}}^{2} e^{-2 \beta T}+3 \frac{(a-2 \gamma) \gamma}{2} \frac{e+1}{2(e-1)} \\
& \times\left\|u_{0}\right\|_{H^{1}}^{4} e^{-4 \beta T}+12 \omega \gamma \sqrt{\frac{e+1}{2(e-1)}}\left\|u_{0}\right\|_{H^{1}}^{3} e^{-3 \beta T} .
\end{aligned}
$$

Setting

$$
\begin{gathered}
m(t)=\int_{\mathbb{S}} \gamma u_{x}^{3} d x+\beta\left\|u_{0}\right\|_{H^{1}}^{2} e^{-2 \beta T} \\
K=\frac{3 \beta^{2}}{2}\left\|u_{0}\right\|_{H^{1}}^{2} e^{-2 \beta T}+3 \frac{(a-2 \gamma) \gamma}{2} \frac{e+1}{2(e-1)}\left\|u_{0}\right\|_{H^{1}}^{4} e^{-4 \beta T} \\
+12 \omega \gamma \sqrt{\frac{e+1}{2(e-1)}}\left\|u_{0}\right\|_{H^{1}}^{3} e^{-3 \beta T}
\end{gathered}
$$

we have

$$
\frac{d}{d t} m(t) \leq-\frac{3}{2\left\|u_{0}\right\|_{H^{1}}^{2} e^{-2 \beta T}} m^{2}(t)+K .
$$

Note that, from the lemma, if $m(0)$ $-\sqrt{2 K / 3}\left\|u_{0}\right\|_{H^{1}} e^{-\beta T}$, similar to the proof in case (i) of Theorem 11, we conclude that the corresponding solution will blow up in finite time.

(ii) The second is $a / 3<\gamma \leq a$; from case (ii) of the theorem, we have $\gamma(3 \gamma-a) \geq 0$ and $G *\left((\gamma(3 \gamma-a) / 4) u_{x}^{2}\right) \geq 0$.

Using (76) and (75), it yields

$$
\begin{aligned}
\frac{d}{d t} \int_{\mathbb{S}} \gamma u_{x}^{3} d x \leq & -\frac{3}{2\left\|u_{0}\right\|_{H^{1}}^{2} e^{-2 \beta T}} \\
& \times\left(\int_{\mathbb{S}} \gamma u_{x}^{3} d x+\beta\left\|u_{0}\right\|_{H^{1}}^{2} e^{-2 \beta T}\right)^{2}+\frac{3 \beta^{2}}{2} \\
& \times\left\|u_{0}\right\|_{H^{1}}^{2} e^{-2 \beta T}+3 \frac{(a-\gamma) \gamma}{4} \frac{e+1}{2(e-1)}\left\|u_{0}\right\|_{H^{1}}^{4} \\
& \times e^{-4 \beta T}+12 \omega \gamma \sqrt{\frac{e+1}{2(e-1)}}\left\|u_{0}\right\|_{H^{1}}^{3} e^{-3 \beta T}
\end{aligned}
$$

Setting

$$
\begin{gathered}
m(t)=\int_{\mathbb{S}} \gamma u_{x}^{3} d x+\beta\left\|u_{0}\right\|_{H^{1}}^{2} e^{-2 \beta T} \\
K=\frac{3 \beta^{2}}{2}\left\|u_{0}\right\|_{H^{1}}^{2} e^{-2 \beta T}+3 \frac{(a-\gamma) \gamma}{4} \frac{e+1}{2(e-1)}\left\|u_{0}\right\|_{H^{1}}^{4} e^{-4 \beta T} \\
+12 \omega \gamma \sqrt{\frac{e+1}{2(e-1)}}\left\|u_{0}\right\|_{H^{1}}^{3} e^{-3 \beta T},
\end{gathered}
$$

we have

$$
\frac{d}{d t} m(t) \leq-\frac{3}{2\left\|u_{0}\right\|_{H^{1}}^{2} e^{-2 \beta T}} m^{2}(t)+K
$$

Note that, from the lemma, if $m(0)<$ $-\sqrt{2 K / 3}\left\|u_{0}\right\|_{H^{1}} e^{-\beta T}$, similar to the proof in case (i) of Theorem 11, we derive that the corresponding solution will blow up in finite time.

(iii) The third is $\gamma>a$ or $\gamma<0$; note that $(a-\gamma) \gamma / 2<0$. Using (76) and (75), it yields

$$
\begin{aligned}
\frac{d}{d t} \int_{\mathbb{S}} \gamma u_{x}^{3} d x \leq & -\frac{3}{2\left\|u_{0}\right\|_{H^{1}}^{2} e^{-2 \beta T}} \\
& \times\left(\int_{\mathbb{S}} \gamma u_{x}^{3} d x+\beta\left\|u_{0}\right\|_{H^{1}}^{2} e^{-2 \beta T}\right)^{2}+\frac{3 \beta^{2}}{2} \\
& \times\left\|u_{0}\right\|_{H^{1}}^{2} e^{-2 \beta T}+3 \frac{(\gamma-a) \gamma}{4} \frac{e+1}{2(e-1)}\left\|u_{0}\right\|_{H^{1}}^{4} \\
& \times e^{-4 \beta T}+12 \omega|\gamma| \sqrt{\frac{e+1}{2(e-1)}}\left\|u_{0}\right\|_{H^{1}}^{3} e^{-3 \beta T}
\end{aligned}
$$

Setting

$$
\begin{gathered}
m(t)=\int_{\mathbb{S}} \gamma u_{x}^{3} d x+\beta\left\|u_{0}\right\|_{H^{1}}^{2} e^{-2 \beta T}, \\
K=\frac{3 \beta^{2}}{2}\left\|u_{0}\right\|_{H^{1}}^{2} e^{-2 \beta T}+3 \frac{(\gamma-a) \gamma}{4} \frac{e+1}{2(e-1)} \\
\times\left\|u_{0}\right\|_{H^{1}}^{4} e^{-4 \beta T}+12 \omega|\gamma| \\
\quad \times \sqrt{\frac{e+1}{2(e-1)}}\left\|u_{0}\right\|_{H^{1}}^{3} e^{-3 \beta T},
\end{gathered}
$$

we have

$$
\frac{d}{d t} m(t) \leq-\frac{3}{2\left\|u_{0}\right\|_{H^{1}}^{2} e^{-2 \beta T}} m^{2}(t)+K .
$$

Note that, from the lemma, if $m(0)$ $-\sqrt{2 K / 3}\left\|u_{0}\right\|_{H^{1}} e^{-\beta T}$, similar to the proof in case (i) of Theorem 11, we deduce that the corresponding solution will blow up in finite time. It completes the proof of Theorem 13. 
Finally, we give the fourth blow-up result.

Theorem 14. Let $\beta \geq 0$ and $u_{0} \in H^{s}, s \geq 3$. Assume that $u_{0} \neq 0$ and $\int_{\mathbb{S}} u_{0} d x=0$. If $\gamma$ and $\omega$ satisfy one of the following conditions:

(i) $a \sinh (1 / 2) /(6+\sinh (1 / 2))<\gamma \leq a$ and $\omega<$ $(6 \sqrt{3} / 169)(3 \gamma / \sinh (1 / 2)-(a-\gamma) / 2)\left\|u_{0}\right\|_{H^{1}} e^{-4 \beta T}$,

(ii) $\gamma>a$ and $\omega<(6 \sqrt{3} / 169)(3 \gamma / \sinh (1 / 2)+(a-$ $\gamma) / 2)\left\|u_{0}\right\|_{H^{1}} e^{-4 \beta T}$

(iii) $\gamma<a \sinh (1 / 2) /(\sinh (1 / 2)-6)$ and $\omega<$ $(6 \sqrt{3} / 169|\gamma|)\left(3 \gamma^{2} / \sinh (1 / 2)+\gamma(a-\gamma) / 2\right)\left\|u_{0}\right\|_{H^{1}} e^{-4 \beta T}$,

then the corresponding solution of (2) blows up in finite time.

Proof. Supposing that the statement is not correct, then the solution of (2) exists globally in time.

Thus, applying $\gamma u_{x}^{2} \partial_{x}$ to both sides of (4) and integrating by parts, we get

$$
\begin{aligned}
\frac{d}{d t} \int_{\mathbb{S}} \gamma u_{x}^{3} d x= & -\frac{3 \gamma^{2}}{2} \int_{\mathbb{S}} u_{x}^{4}-3 \beta \int_{\mathbb{S}} \gamma u_{x}^{3} d x+3 \frac{(a-\gamma) \gamma}{2} \\
& \times \int_{\mathbb{S}} u_{x}^{2} u^{2} d x+6 \omega \gamma \int_{\mathbb{S}} u_{x}^{2} u d x \\
& -3 \int_{\mathbb{S}} u_{x}^{2} G * \frac{(a-\gamma) \gamma}{2} u^{2} d x \\
& -3 \int_{\mathbb{S}} u_{x}^{2} G * \frac{\gamma^{2}}{2} u_{x}^{2} d x-6 \omega \gamma \int_{\mathbb{S}} u_{x}^{2} G * u d x
\end{aligned}
$$

Due to $\|u\|_{L^{\infty}}^{2} \leq(1 / 12) \int_{S} u_{x}^{2} d x \leq(1 / 12)\|u\|_{H^{1}}^{2}$ and $\int_{S} u_{x}^{2} d x \geq(12 / 13)\|u\|_{H^{1}}^{2}$ (see [29]), we get

$$
\begin{gathered}
\int_{\mathbb{S}} u^{2} u_{x}^{2} d x \leq \max \left\{u^{2}\right\} \int_{\mathbb{S}} u_{x}^{2} d x \leq \frac{1}{12}\left(\int_{\mathbb{S}} u_{x}^{2} d x\right)^{2}, \\
\int_{\mathbb{S}} u u_{x}^{2} d x \leq \max \{|u|\} \int_{\mathbb{S}} u_{x}^{2} d x \leq \frac{\sqrt{3}}{6}\left(\int_{\mathbb{S}} u_{x}^{2} d x\right)^{3 / 2}, \\
\int_{\mathbb{S}} u_{x}^{2} G *\left(u^{2}\right) d x \leq\|G\|_{L^{1}} \max \left\{u^{2}\right\} \\
\times \int_{\mathbb{S}} u_{x}^{2} d x \leq \frac{1}{12}\left(\int_{\mathbb{S}} u_{x}^{2} d x\right)^{2}, \\
\int_{\mathbb{S}} u_{x}^{2} G * u d x \leq\|G\|_{L^{1}} \max \{u\} \int_{\mathbb{S}} u_{x}^{2} d x \leq \frac{\sqrt{3}}{6}\left(\int_{\mathbb{S}} u_{x}^{2} d x\right)^{3 / 2} .
\end{gathered}
$$

Since $1 / 2 \sinh (1 / 2) \leq G(x) \leq \cosh (1 / 2) / 2 \sinh (1 / 2)$, we have

$$
\begin{aligned}
\int_{\mathbb{S}} u_{x}^{2} G *\left(u_{x}^{2}\right) d x & \geq \frac{1}{2 \sinh (1 / 2)}\left(\int_{\mathbb{S}} u_{x}^{2} d x\right)^{2} \\
& \geq \frac{72}{169 \sinh (1 / 2)}\|u\|_{H^{1}}^{4} .
\end{aligned}
$$

Therefore, we deduce

$$
\begin{aligned}
\frac{d}{d t} \int_{\mathbb{S}} \gamma u_{x}^{3} d x= & -\frac{3 \gamma^{2}}{2} \int_{\mathbb{S}} u_{x}^{4}-3 \beta \int_{\mathbb{S}} \gamma u_{x}^{3} d x+2 \sqrt{3} \omega|\gamma| \\
& \times\left(\int_{\mathbb{S}} u_{x}^{2} d x\right)^{3 / 2} \\
& +\left(\frac{(a-\gamma) \gamma}{8}-\frac{3 \gamma^{2}}{4 \sinh (1 / 2)}\right)\left(\int_{\mathbb{S}} u_{x}^{2} d x\right)^{2} .
\end{aligned}
$$

Next, we divide (90) three cases to prove the theorem.

(i) The first is $a \sinh (1 / 2) /(6+\sinh (1 / 2))<\gamma \leq a$. Note that $\gamma(a-\gamma) / 8>0$ and $\gamma(a-\gamma) / 8-3 \gamma^{2} / 4 \sinh (1 / 2)<0$. From the condition of Theorem 14, we have

$$
\begin{aligned}
\frac{d}{d t} \int_{\mathbb{S}} \gamma u_{x}^{3} d x \leq & -\frac{3 \gamma^{2}}{2} \int_{\mathbb{S}} u_{x}^{4}-3 \beta \int_{\mathbb{S}} \gamma u_{x}^{3} d x+2 \sqrt{3} \omega \gamma \\
& \times\left\|u_{0}\right\|_{H^{1}}^{3}+\frac{36}{169}\left(\frac{(a-\gamma) \gamma}{2}-\frac{3 \gamma^{2}}{\sinh (1 / 2)}\right) \\
& \times\left\|u_{0}\right\|_{H^{1}}^{4} e^{-4 \beta T} \\
\leq & -\frac{3 \gamma^{2}}{2} \int_{\mathbb{S}} u_{x}^{4}-3 \beta \int_{\mathbb{S}} \gamma u_{x}^{3} d x-\delta,
\end{aligned}
$$

where $\delta=2 \sqrt{3} \omega \gamma\left\|u_{0}\right\|_{H^{1}}^{3}+(36 / 169)((a-\gamma) \gamma / 2-$ $\left.3 \gamma^{2} / \sinh (1 / 2)\right)\left\|u_{0}\right\|_{H^{1}}^{4} e^{-4 \beta T}>0$. From Holder's inequality, we obtain

$$
\int_{\mathbb{S}} \gamma u_{x}^{3} d x \leq \gamma\left(\int_{\mathbb{S}} u_{x}^{3} d x\right)^{3 / 4}
$$

Thus, we have

$$
\frac{d}{d t} \int_{\mathbb{S}} \gamma u_{x}^{3} d x \leq-\frac{3 \gamma^{2 / 3}}{2}\left(\int_{\mathbb{S}} \gamma u_{x}^{3} d x\right)^{4 / 3}-3 \beta \int_{\mathbb{S}} \gamma u_{x}^{3} d x-\delta .
$$

From (93), we have

$$
\frac{d}{d t} \int_{\mathbb{S}} \gamma u_{x}^{3} d x \leq-3 \beta \int_{\mathbb{S}} \gamma u_{x}^{3} d x-\delta .
$$

Solving (94), we obtain

$$
\int_{\mathbb{S}} \gamma u_{x}^{3} d x \leq-\frac{\delta}{3 \beta}+C e^{-3 \beta t}
$$

where $C<0$ is constant which implies that there is a $t_{0} \geq 0$ such that

$$
\int_{\mathbb{S}} \gamma u_{x}^{3} d x<0, \quad t \geq t_{0} .
$$

On the other hand,

$$
\frac{d}{d t} \int_{\mathbb{S}} \gamma u_{x}^{3} d x \leq-\frac{3 \gamma^{2 / 3}}{2}\left(\int_{\mathbb{S}} \gamma u_{x}^{3} d x\right)^{4 / 3}-3 \beta \int_{\mathbb{S}} \gamma u_{x}^{3} d x
$$


Solving (97), we obtain

$$
0>\left(\int_{\mathbb{S}} \gamma u_{x}^{3} d x\right)^{-1} \geq\left(-\frac{\gamma^{2 / 3}}{2 \beta}+C_{1} e^{\beta t}\right)^{3},
$$

where $C_{1} \geq 0$ is constant, which leads to a contradiction as $t$ becomes large enough. Therefore, case (i) is proved.

(ii) The second is $\gamma \geq a$. Note that $\gamma(a-\gamma) / 8<0$ and $-\gamma(a-\gamma) / 8-3 \gamma^{2} / 4 \sinh (1 / 2)<0$. From the condition of the theorem, we have

$$
\begin{aligned}
\frac{d}{d t} \int_{\mathbb{S}} \gamma u_{x}^{3} d x \leq & -\frac{3 \gamma^{2}}{2} \int_{\mathbb{S}} u_{x}^{4}-3 \beta \int_{\mathbb{S}} \gamma u_{x}^{3} d x+2 \sqrt{3} \omega \gamma \\
& \times\left\|u_{0}\right\|_{H^{1}}^{3}+\frac{36}{169}\left(-\frac{(a-\gamma) \gamma}{2}-\frac{3 \gamma^{2}}{\sinh (1 / 2)}\right) \\
& \times\left\|u_{0}\right\|_{H^{1}}^{4} e^{-4 \beta T} \\
\leq & -\frac{3 \gamma^{2}}{2} \int_{\mathbb{S}} u_{x}^{4}-3 \beta \int_{\mathbb{S}} \gamma u_{x}^{3} d x-\delta,
\end{aligned}
$$

where $\delta=2 \sqrt{3} \omega \gamma\left\|u_{0}\right\|_{H^{1}}^{3}+(36 / 169)(-(a-\gamma) \gamma / 2-$ $\left.3 \gamma^{2} / \sinh (1 / 2)\right)\left\|u_{0}\right\|_{H^{1}}^{4} e^{-4 \beta T}>0$. Repeating the arguments in the proof of case (i), we deduce that the corresponding solution blows up.

(iii) The third is $\gamma<a \sinh (1 / 2) /(\sinh (1 / 2)-6)$. Note that $\gamma(a-\gamma) / 8<0$ and $-\gamma(a-\gamma) / 8-3 \gamma^{2} / 4 \sinh (1 / 2)<0$. From the condition of the theorem, we have

$$
\begin{aligned}
\frac{d}{d t} \int_{\mathbb{S}} \gamma u_{x}^{3} d x \leq & -\frac{3 \gamma^{2}}{2} \int_{S} u_{x}^{4}-3 \beta \int_{\mathbb{S}} \gamma u_{x}^{3} d x+2 \sqrt{3} \omega|\gamma| \\
& \times\left\|u_{0}\right\|_{H^{1}}^{3}+\frac{36}{169}\left(-\frac{(a-\gamma) \gamma}{2}-\frac{3 \gamma^{2}}{\sinh (1 / 2)}\right) \\
& \times\left\|u_{0}\right\|_{H^{1}}^{4} e^{-4 \beta T} \\
\leq & -\frac{3 \gamma^{2}}{2} \int_{\mathbb{S}} u_{x}^{4}-3 \beta \int_{\mathbb{S}} \gamma u_{x}^{3} d x-\delta,
\end{aligned}
$$

where $\delta=2 \sqrt{3} \omega|\gamma|\left\|u_{0}\right\|_{H^{1}}^{3}+(36 / 169)(-(a-\gamma) \gamma / 2-$ $\left.3 \gamma^{2} / \sinh (1 / 2)\right)\left\|u_{0}\right\|_{H^{1}}^{4} e^{-4 \beta T}>0$. Repeating the arguments in the proof of case (i), we derive that the corresponding solution blows up. This finishes the proof of the theorem.

\section{Conflict of Interests}

The authors declare that there is no conflict of interests regarding the publication of this paper.

\section{Acknowledgments}

The authors thank the referees for their valuable comments and suggestions. This work is supported by the Key Project of Sichuan Province University Key Laboratory no. 2013QZJ02 and the Research Foundation of the SUSE no. 2011KY12, no. 2011RC10, and no. 2012KY09.

\section{References}

[1] Q. Hu and Z. Yin, "Blowup phenomena for a new periodic nonlinearly dispersive wave equation," Mathematische Nachrichten, vol. 283, no. 11, pp. 1613-1628, 2010.

[2] Z. Yin, "Well-posedness, global solutions and blowup phenomena for a nonlinearly dispersive wave equation," Journal of Evolution Equations, vol. 4, no. 3, pp. 391-419, 2004.

[3] T. B. Benjamin, J. L. Bona, and J. J. Mahony, "Model equations for long waves in nonlinear dispersive systems," Philosophical Transactions of the Royal Society of London. Series A, vol. 272, no. 1220 , pp. $47-78,1972$.

[4] P. Souganidis and W. A. Strauss, "Instability of a class of dispersive solitary waves," Proceedings of the Royal Society of Edinburgh: Section A, vol. 114, no. 3-4, pp. 195-212, 1990.

[5] R. K. Dodd, J. C. Eilbeck, J. D. Gibbon, and H. C. Morris, Solitons and Nonlinear Wave Equations, Academic Press, New York, NY, USA, 1984.

[6] R. Camassa and D. D. Holm, "An integrable shallow water equation with peaked solitons," Physical Review Letters, vol. 71, no. 11, pp. 1661-1664, 1993.

[7] A. Constantin, "The Hamiltonian structure of the CamassaHolm equation," Expositiones Mathematicae, vol. 15, no. 1, pp. 53-85, 1997.

[8] B. Fuchssteiner and A. S. Fokas, "Symplectic structures, their Bäcklund transformations and hereditary symmetries," Physica $D$, vol. 4, no. 1, pp. 47-66, 1981.

[9] J. Lenells, "Conservation laws of the Camassa-Holm equation," Journal of Physics A, vol. 38, no. 4, pp. 869-880, 2005.

[10] A. Constantin, "On the scattering problem for the CamassaHolm equation," Proceedings of the Royal Society A, vol. 457, no. 2008, pp. 953-970, 2001.

[11] A. Constantin and B. Kolev, "Geodesic flow on the diffeomorphism group of the circle," Commentarii Mathematici Helvetici, vol. 78, no. 4, pp. 787-804, 2003.

[12] A. Constantin, T. Kappeler, B. Kolev, and P. Topalov, "On geodesic exponential maps of the Virasoro group," Annals of Global Analysis and Geometry, vol. 31, no. 2, pp. 155-180, 2007.

[13] A. Constantin and J. Escher, "Global existence and blow-up for a shallow water equation," Annali della Scuola Normale Superiore di Pisa, vol. 26, no. 2, pp. 303-328, 1998.

[14] Y. A. Li and P. J. Olver, "Well-posedness and blow-up solutions for an integrable nonlinearly dispersive model wave equation," Journal of Differential Equations, vol. 162, no. 1, pp. 27-63, 2000.

[15] G. Rodríguez-Blanco, "On the Cauchy problem for the Camassa-Holm equation," Nonlinear Analysis: Theory, Methods \& Applications, vol. 46, no. 3, pp. 309-327, 2001.

[16] A. Constantin, "Existence of permanent and breaking waves for a shallow water equation: a geometric approach," Annales de l'Institut Fourier, vol. 50, no. 2, pp. 321-362, 2000.

[17] A. Constantin and J. Escher, "Wave breaking for nonlinear nonlocal shallow water equations," Acta Mathematica, vol. 181, no. 2, pp. 229-243, 1998.

[18] A. Constantin, "On the blow-up of solutions of a periodic shallow water equation," Journal of Nonlinear Science, vol. 10, no. 3, pp. 391-399, 2000.

[19] A. Constantin and H. P. McKean, "A shallow water equation on the circle," Communications on Pure and Applied Mathematics, vol. 52, no. 8, pp. 949-982, 1999.

[20] Y. Zhou, "Wave breaking for a shallow water equation," Nonlinear Analysis: Theory, Methods \& Applications, vol. 57, no. 1, pp. 137-152, 2004. 
[21] E. Wahlén, "On the blow-up of solutions to the periodic Camassa-Holm equation," Nonlinear Differential Equations and Applications, vol. 13, no. 5-6, pp. 643-653, 2007.

[22] Z. Yin, "On the blow-up of solutions of the periodic camassaholm equation," Dynamics of Continuous, Discrete and Impulsive Systems Series A, vol. 12, no. 3-4, pp. 375-381, 2005.

[23] A. Constantin and L. Molinet, "Global weak solutions for a shallow water equation," Communications in Mathematical Physics, vol. 211, no. 1, pp. 45-61, 2000.

[24] Z. Xin and P. Zhang, "On the weak solutions to a shallow water equation," Communications on Pure and Applied Mathematics, vol. 53, no. 11, pp. 1411-1433, 2000.

[25] Z. Xin and P. Zhang, "On the uniqueness and large time behavior of the weak solutions to a shallow water equation," Communications in Partial Differential Equations, vol. 27, no. 910, pp. 1815-1844, 2002.

[26] H.-H. Dai, "Model equations for nonlinear dispersive waves in a compressible Mooney-Rivlin rod," Acta Mechanica, vol. 127, no. 1-4, pp. 193-207, 1998.

[27] A. Constantin and W. A. Strauss, "Stability of a class of solitary waves in compressible elastic rods," Physics Letters A, vol. 270, no. 3-4, pp. 140-148, 2000.

[28] Y. Zhou, "Local well-posedness and blow-up criteria of solutions for a rod equation," Mathematische Nachrichten, vol. 278, no. 14, pp. 1726-1739, 2005.

[29] Y. Zhou, "Blow-up phenomenon for a periodic rod equation," Physics Letters A, vol. 353, no. 6, pp. 479-486, 2006.

[30] Z. Yin, "On the blow-up of solutions of a periodic nonlinear dispersive wave equation in compressible elastic rods," Journal of Mathematical Analysis and Applications, vol. 288, no. 1, pp. 232-245, 2003.

[31] S. Wu and Z. Yin, "Global existence and blow-up phenomena for the weakly dissipative Camassa-Holm equation," Journal of Differential Equations, vol. 246, no. 11, pp. 4309-4321, 2009.

[32] T. Kato and G. Ponce, "Commutator estamaes and the Euler and Navier-Stoks equations," Communications on Pure and Applied Mathematics, vol. 41, pp. 891-907, 1998. 


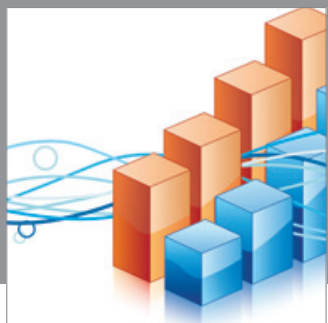

Advances in

Operations Research

mansans

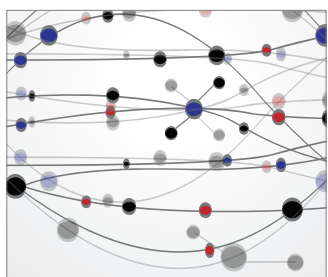

The Scientific World Journal
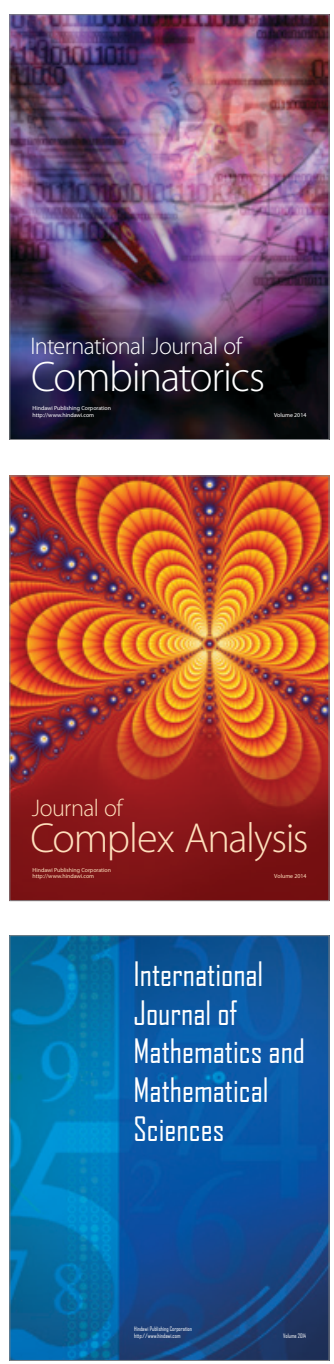
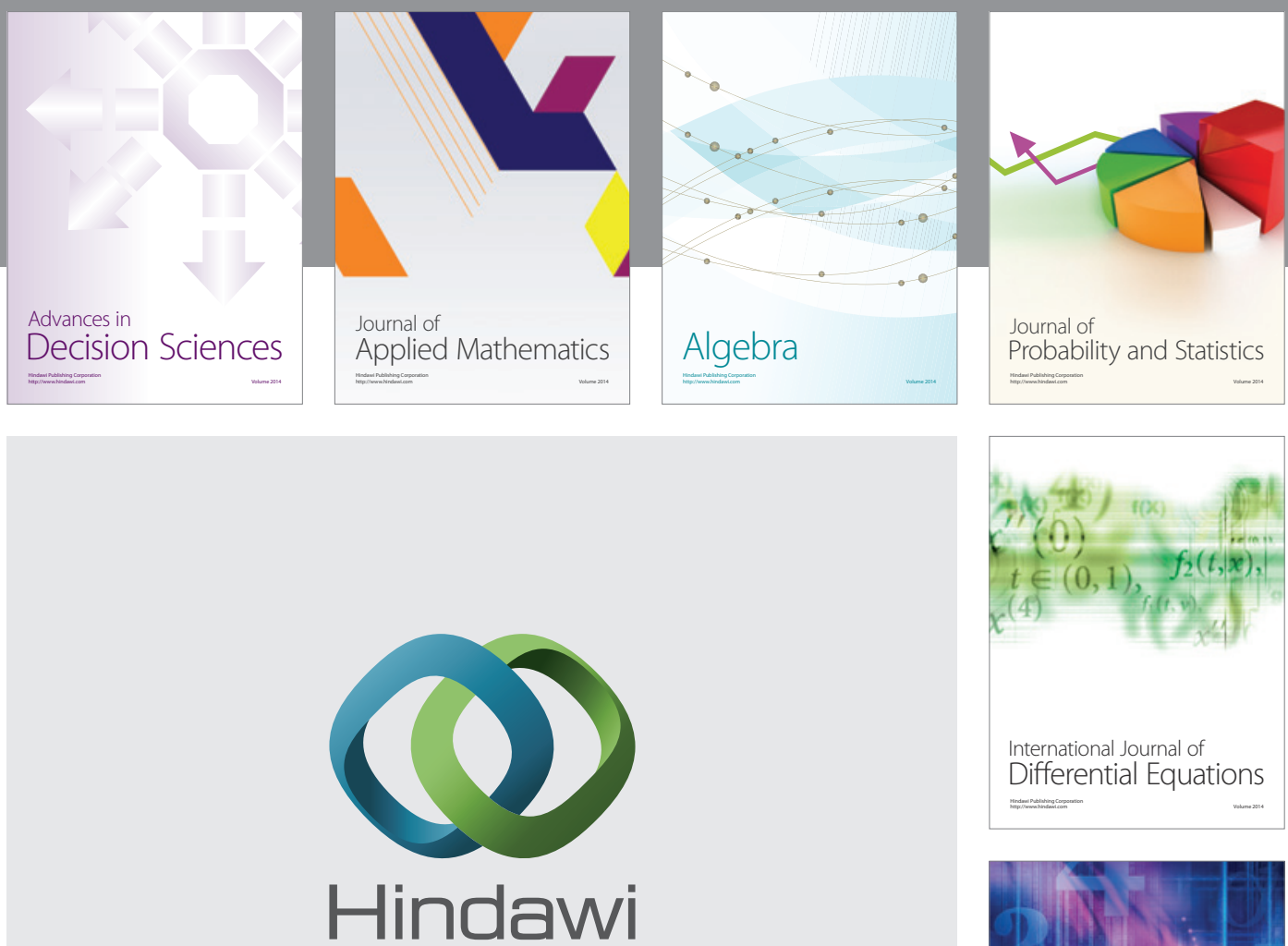

Submit your manuscripts at http://www.hindawi.com
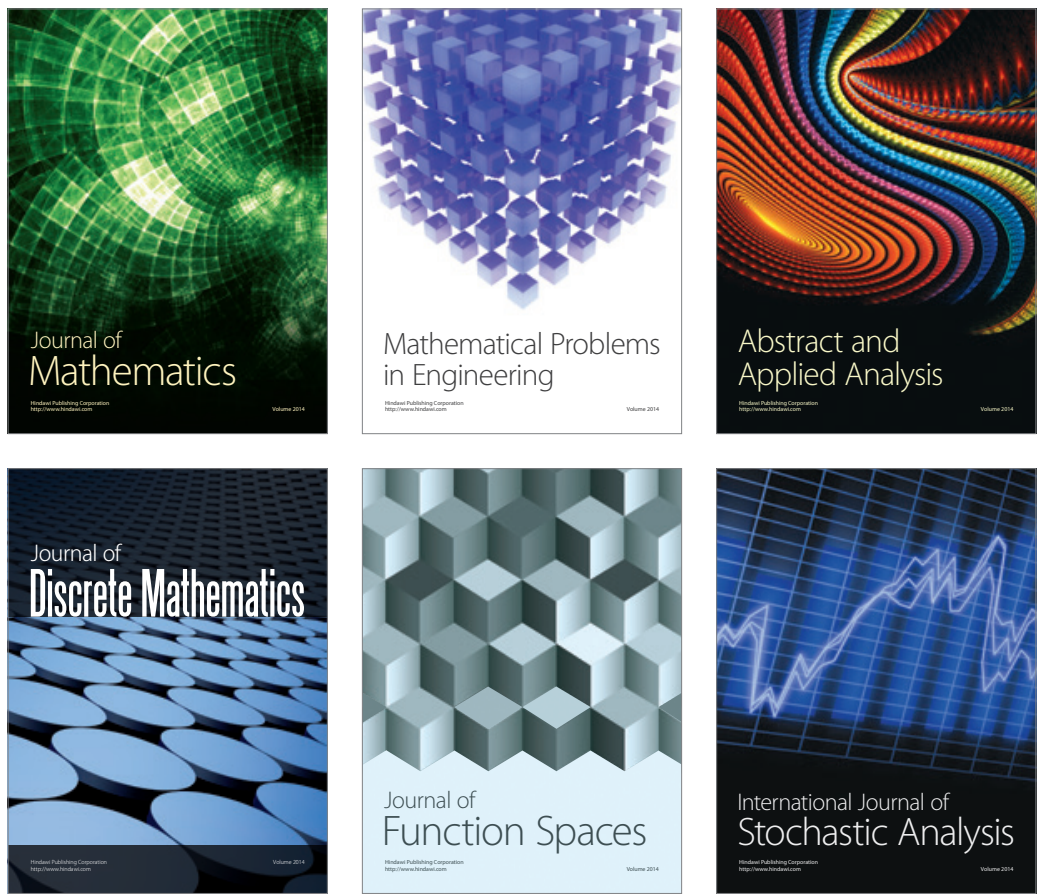

Journal of

Function Spaces

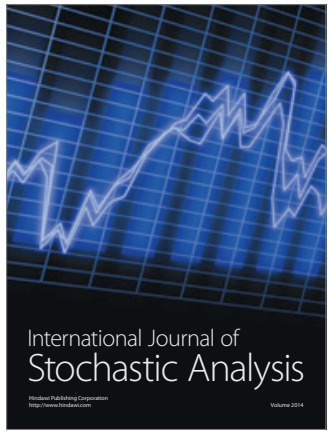

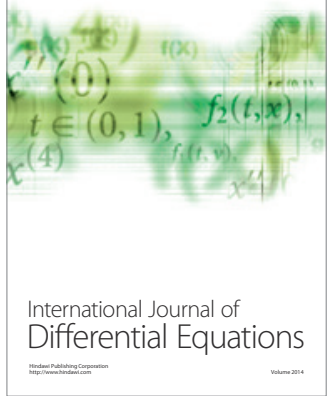
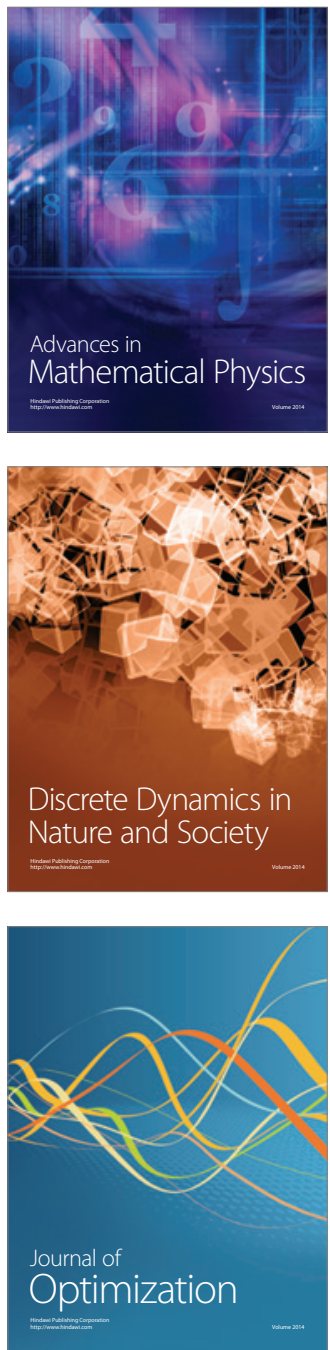